\section{Toward scalable in vitro models: a novel experimental and computational pipeline for the identification of cellular metabolic parameters}

\author{
Piera Mancini, ${ }^{1}$ Ermes Botte, 1,2 \\ Chiara Magliaro, ${ }^{1}$ Arti Ahluwalia1,2 \\ 1Research Center "E. Piaggio",
} University of Pisa, Pisa; ${ }^{2}$ Department of Information Engineering, University of Pisa, Pisa, Italy

\begin{abstract}
Oxygen utilization by cells has a crucial role in the design of advanced in vitro models. The aim of this study is to develop an experimental and computational pipeline for identifying oxygen metabolism parameters. We applied the approach to HepG2 cell monolayer cultures, demonstrating that such parameters depend on cell density.
\end{abstract}

\section{Introduction}

Size-dependent scaling of oxygen metabolic rate has been recently introduced as a reliable testbed for the translational power of in vitro cell aggregates. ${ }^{1}$ In fact, oxygen is a key player in cellular respiration and has a major influence on cell growth and fate. However, its low solubility in culture media may result in an inadequate supply, limiting the perspective of $3 \mathrm{D}$ cell cultures. ${ }^{2}$ Generally, oxygen consumption is described by the Michaelis-Menten's (MM) equation:

$$
\begin{aligned}
& R(x, y, z, t) \\
& =-\rho_{c} O C R \frac{C(x, y, z, t)}{k_{M}+C(x, y, z, t)}
\end{aligned}
$$

where $\mathrm{C}\left[\mathrm{mol} \mathrm{m}^{-3}\right]$ is the oxygen concentration, $\rho_{c}\left[\right.$ cell $\left.\mathrm{m}^{-3}\right]$ is the cell density, OCR $\left[\mathrm{mol} \mathrm{cell-1} \mathrm{s}^{-1}\right]$ is the average oxygen consumption rate, and $\mathrm{k}_{\mathrm{M}}\left[\mathrm{mol} \mathrm{m}^{-3}\right]$ is the $\mathrm{MM}$ constant. OCR and $\mathrm{k}_{\mathrm{M}}$ are kinetic parameters assuming values specific for the cell type and have been usually intended as constants. Although, Magliaro et al. have recently shown their dependence on cell density in $3 \mathrm{D}$ in vitro constructs, ${ }^{3}$ estimating consumption parameters by optimizing the MM model fitting on experimental measurements of surface oxygen concentrations at the bottom of the constructs. To determine if the same behaviour is also manifested in monolayers, we developed an experimental and computational pipeline for identifying OCR and $\mathrm{k}_{\mathrm{M}}$ values in $2 \mathrm{D}$ cultures varying cell density, as summarized in Figure 1.

\section{Materials and Methods}

Experimental measurements. Experiments were performed using an oxygen sensor spot (OXSP5, Pyroscience $\mathrm{GmbH}$ ) attached to the bottom of a well of a 96-well plate, placed in a temperaturecontrolled chamber. Before HepG2 cell seeding (Table 1), the sensors were coated with gelatin to promote cell adhesion. At the beginning of each experiment, a two-point calibration was carried out for establishing the level of $100 \%$ oxygen saturation and the totally hypoxic condition. Furthermore, the initial condition was set at $100 \%$ oxygen saturation by dispensing fresh culture medium to the monolayer, while the upper boundary was rendered oxygen impermeable by combining the use of a layer of mineral oil and an oxygen consuming chemical filter (ThermoFisher Scientific). In this configuration, we acquired the average oxygen concentration at the cell surface until steady state was reached, repeating the experiment at least in triplicate for each cell density.

Parameter identification. The in vitro experiments were "replicated" in silico, simulating the dynamics of the system in Matlab (R2020b, The MathWorks, Inc). Given the cylindrical geometry of the system and assuming that cells are homogeneously distributed on the bottom of the well, oxygen dynamics can be assumed as dependent on the axial direction only. The system is physically governed by oxygen transport and consumption described by the second Fick's law (Eq. 2):

$\frac{\partial C(z, t)}{\partial t}=D \frac{\partial^{2} C(z, t)}{\partial z^{2}}$

and by the following initial (Eq. 3) and boundary (Eq. 4, 5) conditions:

$$
\begin{aligned}
& C(z, 0)=C_{0} \\
& J_{C}(H, t)=0 \\
& J_{c}(0, t)=-\rho_{c} O C R \frac{C(0, t)}{k_{M}+C(0, t)}
\end{aligned}
$$

\section{Discussion and Conclusions}

This study defines an experimental and

Correspondence: Piera Mancini, Research Center "E. Piaggio", University of Pisa, Pisa, Italy.

E-mail: p.mancini1@studenti.unipi.it

Key words: In vitro models; 2D cell cultures; oxygen consumption rate; parameter identification.

Acknowledgments: The work is supported by the SnF SINERGIA Project (2019 572 CRSII5_186422/1).

Disclosures: The authors declare no conflicts of interest.

Conference presentation: This paper was presented at the Third Centro 3R Annual Meeting - L'era delle 3R: modelli in silico, in vitro e in vivo per promuovere la ricerca traslazionale 30 September - 1 October 2021, Evento online organizzato dal Politecnico di Torino.

Received for publication: 9 July 2021.

Accepted for publication: 7 September 2021.

This work is licensed under a Creative Commons Attribution NonCommercial 4.0 License (CC BY-NC 4.0).

CC Copyright: the Author(s), 2021

Licensee PAGEPress, Italy

Biomedical Science and Engineering 2021; $4($ s1):179 doi:10.4081/bse.2021.179

where $\mathrm{H}[\mathrm{m}]$ is the culture medium height, $\mathrm{C}_{0}\left[\mathrm{~mol} \mathrm{~m}^{-3}\right]$ is the oxygen concentration in saturated culture medium and $\mathrm{J}_{\mathrm{c}}\left[\mathrm{mol} \mathrm{m}^{-2} \mathrm{~s}^{-}\right.$ 1] is the oxygen flux modelling the cell layer consumption. OCR values were identified for each $\rho_{c}$ by iteratively minimizing the mean square error between $\mathrm{C}(0, \mathrm{t})$ predicted in silico and the corresponding experimental concentration profile (Figure 1). Statistical analyses were performed using the non-parametric Kruskal-Wallis test and non-linear correlation with respect to cell density (Graph Pad Prism 7).

\section{Results}

The Kruskal-Wallis test highlighted a statistically significant difference between OCRs at different $\rho_{\mathrm{c}}(\mathrm{p}<0.0001)$. Median values are reported in Table 1 .

Also, a significant correlation $\left(\mathrm{R}^{2}=\right.$ 0.6409 ) between OCR and $\rho_{c}$ emerges through the non-linear fitting, as shown in Figure 2. 


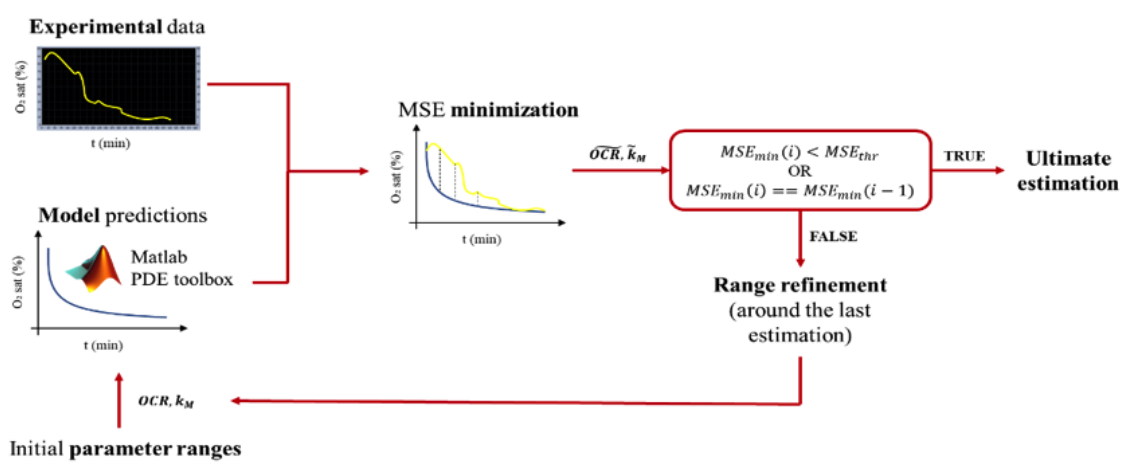

Figure 1. Block diagram illustrating the experimental and computational pipeline developed for identifying kinetic parameters characterizing oxygen metabolism in cell monolayers.

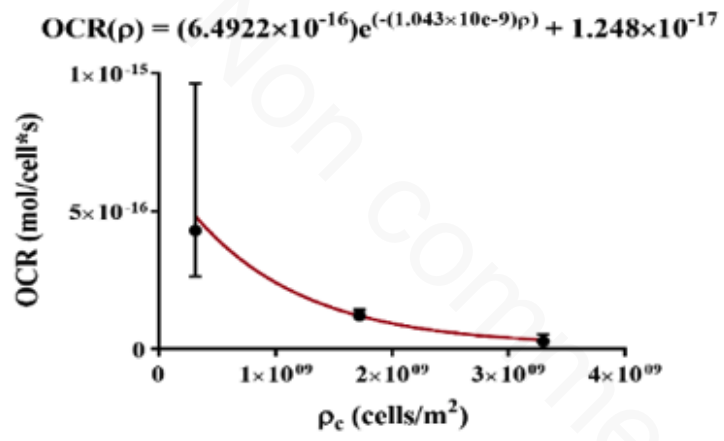

Figure 2. The non-linear dependence of OCR on $\rho \mathrm{c}$ and its analytical formulation as a single-phase decay function. proteome
Table 1. OCR identified for each $\rho c$. The number of experimental measurements $\mathrm{N}$ is also reported.

\begin{tabular}{|c|c|c|}
\hline$\rho_{\mathrm{c}}\left[\right.$ cell m $\left.\mathrm{m}^{-2}\right]$ & OCR $\left[\mathrm{mol} \mathrm{cell}{ }^{-1} \mathbf{s}^{-1}\right]$ & $\mathrm{N}$ \\
\hline $3.10 \times 10^{8}$ & $4.30 \times 10^{-16}$ & 5 \\
\hline $1.72 \times 10^{9}$ & $1.20 \times 10^{-16}$ & 6 \\
\hline $3.30 \times 10^{9}$ & $2.79 \times 10^{-17}$ & 5 \\
\hline
\end{tabular}

computational pipeline allowing the estimation of metabolic parameters and cell density in monolayer cultures. In particular, the approach has been applied to HepG2 cells. The results show that OCR depends on cell density, suggesting cooperative metabolic behaviour when oxygen availability is limited. The same analysis is ongoing for $\mathrm{k}_{\mathrm{M}}$.

\section{References}

1. Ahluwalia A. Allometric scaling invitro. Sci Rep 2017;7:42113.

2. Lovett M, Lee K, Edwards A, Kaplan DL. Vascularization strategies for tissue engineering. Tissue Eng Part B Rev 2009; 15:353-70.

3. Magliaro C, Mattei G, Iacoangeli F, et al. Oxygen consumption characteristics in 3D constructs depend on cell density. Front Bioeng Biotechnol 2019;7:251. 